\title{
Cognitive function and other risk factors for mild traumatic brain injury in young men: nationwide cohort study \\ (9) (1) $\Theta$ OPEN ACCESS
}

\author{
Anna Nordström associate professor ${ }^{1}$, Benoni B Edin professor ${ }^{2}$, Sara Lindström research scientist ${ }^{3}$, \\ Peter Nordström professor ${ }^{4}$
}

1'Department of Community Medicine and Rehabilitation, Rehabilitation Medicine, Umeå University, SE-901 87 Umeå, Sweden; ${ }^{2}$ Department of Integrative Medical Biology, Physiology Section, Umeå University; ${ }^{3}$ Department of Epidemiology, Harvard School of Public Health, Boston, MA, USA; ${ }^{4}$ Department of Community Medicine and Rehabilitation, Geriatric Medicine, Umeå University

\author{
Abstract \\ Objective To investigate cognitive function and other risk factors for \\ mild traumatic brain injury in young men. \\ Design Nationwide prospective cohort study. \\ Setting Sweden
}

Participants 305885 men conscripted for military service from 1989 to 1994.

Main outcome measure mild traumatic brain injuries in relation to cognitive function and other potential risk factors assessed at conscription and follow-up.

Results Men with one mild traumatic brain injury within two years before $(n=1988)$ or after cognitive testing $(n=2214)$ had about $5.5 \%$ lower overall cognitive function scores than did men with no mild traumatic brain injury during follow up $(P<0.001$ for both). Moreover, men with at least two mild traumatic brain injuries after cognitive testing $(n=795)$ had $15 \%$ lower overall cognitive function scores compared with those with no such injury $(P<0.001)$. Independent strong risk factors $\left(P<1 \times 10^{-10}\right)$ for at least one mild traumatic brain injury after cognitive testing ( $n=12494$ events) included low overall cognitive function, a previous mild traumatic brain injury, hospital admission for intoxications, and low education and socioeconomic status. In a sub-cohort of twin pairs in which one twin had a mild traumatic brain injury before cognitive testing $(n=63)$, both twins had lower logical performance and technical performance compared with men in the total cohort with no mild traumatic brain injury $(P<0.05$ for all).

Conclusion Low cognitive function, intoxications, and factors related to low socioeconomic status were strong independent risk factors for mild traumatic brain injuries in men. The low cognitive function in twin pairs discordant for mild traumatic brain injury suggests a genetic component to the low cognitive function associated with such injuries. The study included only men, so inferences to women should be made with caution.

\section{Introduction}

Traumatic brain injury requiring medical attention or resulting in either hospital admission or death has been estimated to affect be more than 10 million people annually worldwide. ${ }^{12}$ Traumatic brain injury is usually characterised as mild, moderate, or severe and is heterogeneous in terms of pathology, severity, diagnostic procedures, treatment, and prognosis. Mild traumatic brain injury is the most common head injury, accounting for approximately $70-90 \%$ of all traumatic brain injuries. Mild traumatic brain injury and concussion are often used interchangeably, although the second term is not well defined in either clinical or research contexts. The annual incidence of mild traumatic brain injury treated in hospital in the general population has been estimated to be $100-300$ per 100000 , and epidemiological data have shown that the incidence is highest among young males. ${ }^{3}$

The literature suggests that mild traumatic brain injuries cause neuropathological changes in brain tissues and have important long term consequences with respect to cognitive function. ${ }^{4-6}$ Cognitive deficits have been described in several areas, including attention, working memory, episodic memory, verbal learning, and processing speed..$^{5-10}$ Such long lasting neuropsychological impairments may occur in $15-25 \%$ of people who have a mild traumatic brain injury. ${ }^{511}{ }^{12}$ Diffusion tensor imaging has shown long lasting signs of abnormalities in the brain more than six months after a blast related mild traumatic brain injury in about $25 \%$ of people investigated. ${ }^{13}$

Nevertheless, the long term cognitive deficits described are based on retrospective data, and little is known about cognitive function before a mild traumatic brain injury in population based cohorts. In a recent study, we found a $7 \%$ lower cognitive function in men who later sustained a traumatic subdural 
hematoma, suggesting that a low cognitive function may be a risk factor for severe traumatic head injuries. ${ }^{14}$ Whether low cognitive function is also a risk factor for the less severe but much more common mild traumatic brain injury is not known. Furthermore, prospective risk factors for mild traumatic brain injuries identified in population based cohorts are lacking.

The aim of this study was to examine cognitive function and other risk factors for mild traumatic brain injuries in a nationwide cohort of Swedish men. We also studied the relation between cognitive function and mild traumatic brain injury in a sub-cohort of twin pairs in which one twin in each pair had sustained a mild traumatic brain injury.

\section{Methods \\ Study population}

Our cohort comprised all Swedish men conscripted for mandatory military service between 1989 and $1994(\mathrm{n}=312$ 908). Of these men, we excluded 7018 (2.2\%) owing to young age $(<16.5$ years at baseline) or with missing age data, extreme body weight $(<40 \mathrm{or}>170 \mathrm{~kg})$ or height $(<140$ or $>215 \mathrm{~cm})$, or missing weight or height data. We also excluded five men who had been conscripted twice. We thus included 305885 (97.8\%) men in these analyses. The background of the Swedish military service conscription register has been described in detail elsewhere. ${ }^{15}$ Until recently, exemptions were granted only for incarcerated men and those with severe chronic medical conditions or disabilities documented by a medical certificate. Accordingly, only about $3 \%$ of all Swedish men were exempted from conscription during the time period considered in this study.

\section{Baseline examination and exclusion criteria}

All conscripts took part in a two day, standardised intelligence and physical examination at six regional conscription centres in Sweden before assignment in the Swedish Armed Forces. Each conscript had a medical examination conducted by a physician, and any identified disorder was classified according to the Swedish version of the ICD-8 (international classification of diseases, 8th revision). Weight, height, hearing, and vision were measured by using standardised methods. Physical fitness was assessed by using an electrically braked bicycle ergometry test as described in detail before. ${ }^{14}{ }^{16} \mathrm{We}$ collected information about total income, disability pension, and highest achieved education 14 years after the conscription tests-that is, at a mean age of 32 years - from the Statistics Sweden database. We classified education into four groups: elementary school only, two years of secondary school (aiming at professional work), three years of secondary school (often theoretical aiming at university studies), and education at university level. Information about full siblings also came from the Statistics Sweden database. We defined twins as two full siblings born the same day. We could not determine whether these twins were monozygotic or dizygotic.

\section{Cognitive function tests}

The tests of cognitive function used during conscription have been described in detail previously. ${ }^{17-19}$ Briefly, the word recollection test assessed knowledge of synonyms (that is, the ability to determine which of four alternatives was the synonym of a given word), the visuospatial geometric perception test assessed the ability to correctly identify a three dimensional object from a series of two dimensional drawings, the logical/inductive performance test assessed the capacity to understand written instructions and apply them to a problem solving task, and the theoretical/technical test assessed mathematical/physics problem solving. The maximum score for the first three tests was 40 points, and that for the theoretical/technical test was 52 points. During the years of conscription (1989-94), cognitive test scores were missing for 32 747, 30 998, 30 474, and 40160 men for the word recollection, visuospatial, logical, and technical tests. Men with missing values were 1 month older at baseline and had a $0.3 \%$ higher risk of a mild traumatic brain injury during follow-up than the rest of the cohort. We converted the test results to normal scores for each year and then expressed them as a score of 0-40 points. The scores of all four tests were significantly correlated ( $r=0.49-0.88 ; \mathrm{P}<0.001$ for all pairwise correlations), and we created a measure of overall cognitive function by summing the $\mathrm{z}$ normalised scores of all tests. We then expressed the overall cognitive function score as a score of 0-40 points. As for previous estimates of general intelligence, ${ }^{20}$ this score was associated with educational achievements $(r=0.55)$ and total income $(r=0.23)$ in this study.

\section{Follow-up data collection}

Information on diagnoses of mild traumatic brain injuries between 1 January 1987 and 31 December 2010 came from the national hospital discharge register: code 850.x (ICD-9; 1987-97) and code S06.0x (ICD-10; 1998-2010). This registry records the underlying cause of an injury but not the severity of the injury. For this study we used all diagnoses of mild traumatic brain injury in further analysis. We assessed information on intoxications (from alcohol or drugs) by using the ICD-9 codes 303.x and 304. $x$ and the ICD-10 codes F10.x, F11.x, and F12.x. The hospital discharge register covers all public inpatient care in Sweden from 1987 and all outpatient care in Sweden from 2001 by using the ICD-9 and ICD-10 codes. Each study participant is identified through his individual unique national registration number, which includes the date of birth. Using this registration number, we achieved follow-up by record linkage to the hospital discharge register. In validation studies, the different diagnoses in the hospital discharge register have a positive predictive value ranging from $85 \%$ to $97 \% .{ }^{21}$ If the interval between two diagnosed mild traumatic brain injuries was more than 179 days, we considered the second injury to be a new mild traumatic brain injury.

We obtained information on deaths occurring during the study period through record linkage with the national cause of death register, administered by the Center for Epidemiology at the National Board of Health and Welfare in Sweden. Information about emigration came from the Statistics Sweden database.

\section{Statistical analyses}

We investigated baseline differences among men with no mild traumatic brain injury, men with a mild traumatic brain injury before cognitive testing, and men who sustained one or more mild traumatic brain injuries after cognitive testing by using analysis of variance with Bonferroni's post hoc correction of $\mathrm{P}$ values. We used the $\chi^{2}$ test to investigate differences in highest achieved education among the four groups and Pearson's correlations coefficient to investigate bivariate associations. We investigated differences between twin pairs by using a paired samples $t$ test. We used logistic regression to test associations between the baseline cognitive function and previous mild traumatic brain injury and Cox proportional hazard models to test associations between baseline cognitive function and later risk of mild traumatic brain injury. For analyses of previous mild traumatic brain injuries, we excluded all men with a mild 
traumatic brain injury after cognitive testing. Similarly, we excluded all men with a mild traumatic brain injury before cognitive testing when studying mild traumatic brain injuries during follow-up. All models were adjusted for age, weight, height, vision, hearing, physical fitness, place and year of conscription, and all diagnoses at baseline as listed in table $1 \Downarrow$. The study endpoint for all Cox regression models was the date of a registered mild traumatic brain injury, date of death, date of emigration, or the end of the study period (31 December 2010), whichever came first. We checked the proportional hazard assumption graphically by using Kaplan-Meier curves. Using logistic regression, we further tested independent risk factors for a mild traumatic brain injury after cognitive testing by also including men with mild traumatic brain injuries before baseline, intoxications diagnosed at hospital admissions after baseline but no later than at time of mild traumatic brain injury, socioeconomic factors, and highest achieved education. We used SPSS software (version 18 for PC) for all statistical analyses. All statistical tests were two sided, and we considered a $\mathrm{P}$ value less than 0.05 to be statistically significant.

\section{Results}

\section{Baseline characteristics}

Our study population included 305885 men with a mean age of 18.3 (SD 0.8) years at baseline (that is, when the cognitive tests were performed). The cohort included 4713 men who had sustained one mild traumatic brain injury before the tests of cognitive function. In the rest of the cohort, 11217 men sustained one mild traumatic brain injury and 795 men sustained at least two such injuries after cognitive testing, during a median follow-up period of 19 (range 0-22) years. The mean hospital stay for those with one mild traumatic brain injury was 1.2 days, and $94 \%$ had a hospital stay of at most two days.

Table $1 \Downarrow$ shows the baseline characteristics according to mild traumatic brain injury status. Compared with men who sustaining no mild traumatic brain injury, the baseline overall cognitive function of men who sustained a mild traumatic brain injury before or after cognitive testing was significantly lower ( $\mathrm{P}<0.001$ for both) (fig $1 \Downarrow$ ). Furthermore, overall cognitive function was $15.1 \%$ lower $(\mathrm{P}<0.001)$ for men who sustained at least two mild traumatic brain injuries after cognitive testing. Length of education was inversely associated with number of mild traumatic brain injuries $(\mathrm{P}<0.001$ for trend), and total income was lower and early disability pension more common especially in men with a mild traumatic brain injury after cognitive testing. Hospital admission for acute intoxications during follow-up was significantly more common in those with one and especially multiple mild traumatic brain injuries during follow-up ( $\mathrm{P}<0.001$ for all comparisons). The most common causes of mild traumatic brain injury were falling $(n=5754)$, trauma during transportation $(n=5739)$, and assault $(n=2433)$.

\section{Cognitive function in men with a mild traumatic brain injury within two years of cognitive testing}

Men who had mild traumatic brain injuries within two years before cognitive testing $(\mathrm{n}=1988)$ had 5.6\% lower overall cognitive function compared with men with no such injury during follow-up. After excluding all men with a mild traumatic brain injury before cognitive testing ( $\mathrm{n}=4713), 2214$ men with a mild traumatic brain injury within two years after cognitive testing had on average $5.3 \%$ lower overall cognitive function compared with those with no such injuries during the follow-up period (fig $1 \Downarrow$ ). To investigate any short term differences in cognitive function, we evaluated men with a mild traumatic brain injury within three months before cognitive testing. These men had similar cognitive function scores to men with a mild traumatic brain injury within two years after the cognitive tests (fig $1 \Downarrow$ ).

The number of men with mild traumatic brain injuries before or after the cognitive tests increased monotonically with decreasing tenths of overall cognitive function $(\mathrm{P}<0.001$ for trend for all; data not shown). Being in the lowest compared with the highest tenth of overall cognitive function was associated with an increased risk of having sustained one mild traumatic brain injury within two years before the cognitive tests (odds ratio $2.08,95 \%$ confidence interval 1.63 to 2.64), after adjustment for confounders (fig $2 \Downarrow$, top panel). Being in the lowest compared with the highest tenth of overall cognitive function was also associated with an increased risk of sustaining one mild traumatic brain injury within two years after the cognitive tests (hazard ratio $2.20,1.76$ to 2.76 ) (fig $2 \Downarrow$, middle panel). Finally, being in the lowest compared with the highest tenth of overall cognitive function increased the risk of sustaining at least two mild traumatic brain injuries after cognitive testing (hazard ratio $8.45,5.22$ to 13.67 ) (fig $2 \Downarrow$, bottom panel).

\section{Cognitive function in twins discordant for mild traumatic brain injuries}

The total cohort included 2287 identified twin pairs. In 63 of these twin pairs, only one twin had a mild traumatic brain injury before cognitive testing. Within these twin pairs, we found no significant difference in any of the measures of cognitive function between the twin who had sustained a mild traumatic brain injury and the other twin (table $2 \Downarrow$ ). Both twins had lower logical performance and technical performance at baseline compared with men who did not sustain a mild traumatic brain injury $(\mathrm{P}<0.05)($ table $2 \Downarrow)$.

\section{Cognitive function and other risk factors for mild traumatic brain injury after cognitive testing}

Figure $3 \Downarrow$ shows the cumulative number of mild traumatic brain injuries after cognitive testing for tenths of overall cognitive function. The 600th mild traumatic brain injury was sustained at an age of 23 years of age in the lowest tenth of overall cognitive function compared with 35 years in the highest tenth. We sought to identify independent risk factors for at least one mild traumatic brain injury ( $\mathrm{n}=12494$ events) after the cognitive function tests. We included all variables in table $1 \Downarrow$ as well as a mild traumatic brain injury before the cognitive function tests in the same logistic regression model. Independent strong risk factors $\left(\mathrm{P}<10^{-10}\right.$ for all $)$ included low overall cognitive function (odds ratio $1.14,1.11$ to 1.17 per standard deviation decrease), low total income (1.14, 1.11 to 1.17 per SD decrease), high physical fitness $(1.15,1.12$ to 1.17 per SD increase), hospital admission for an intoxication $(2.41,2.22$ to 2.61$)$, a previous mild traumatic brain injury $(2.08,1.86$ to 2.33$)$, low education (1.76, 1.62 to 1.91 , comparing university with elementary school), and early disability pension $(1.52,1.34$ to 1.73$)$. Other significant risk factors included poor vision, young age, and low weight $(\mathrm{P}<0.05$ for all).

\section{Discussion}

In this study, we found similar deficits in cognitive function in men with mild traumatic brain injuries before and after cognitive testing. In addition, twin pairs discordant for a mild traumatic 
brain injury before cognitive testing had similar cognitive test scores, indicating a genetic component to the low cognitive function observed. Other risk factors included low education, intoxications, and a low socioeconomic status. Altogether, these results suggest that low cognitive function and factors related to a low socioeconomic status are important risk factors for mild traumatic brain injuries. These results may have implications for both the interpretation and rehabilitation of chronic symptoms following mild traumatic brain injuries, and for successful prevention of new injuries.

\section{Clinical implications of findings}

Previous studies based on retrospective data have found long term consequences of mild traumatic brain injuries on different aspects of cognitive function including attention, working memory, and processing speed. ${ }^{7}{ }^{10}$ A meta-analysis including 312 patients suggested an approximately $5 \%$ deficit in cognitive function more than three months after mild traumatic brain injury. ${ }^{22}$ This result was confirmed in our study for all estimates of cognitive function in men with a mild traumatic brain injury before cognitive testing. Nevertheless, the 11000 men with a mild traumatic brain injury after cognitive testing had about $6 \%$ lower overall cognitive function compared with men with no such injury during follow-up, with significantly lower scores also for the separate tests of cognitive function. Furthermore, men with at least two mild traumatic brain injuries after cognitive testing had about $15 \%$ lower cognitive function many years before the injuries were sustained. Altogether, these results suggest that a low cognitive function may be a risk factor for rather than the long term consequence of mild traumatic brain injury. This could be important to consider when evaluating cognitive function after mild traumatic brain injury, and perhaps especially after multiple such injuries. In athletes active in sports with a high risk of head injuries, the results also underscore the importance of pre-morbid cognitive testing for later comparison in those who sustain mild traumatic brain injuries.

To further evaluate the nature of the low cognitive function associated with mild traumatic brain injuries, we investigated cognitive function in twin pairs in which only one of the twins had a mild traumatic brain injury before cognitive testing. Both twins had lower logical and technical function compared with men in the total cohort with no mild traumatic brain injuries. We also found a strong association between the different cognitive tests within these twin pairs. Given that most of the variation in cognitive function is controlled by genetic factors and that twins share at least $50 \%$ of their genome, ${ }^{23}$ our data indicate that genetic factors and a similar environment explain the lower cognitive function found in these twins. In support of our results, cognitive deficits and structural abnormalities of the brain were found in both twins when only one had been exposed to combat exposure and developed a post-traumatic stress syndrome. ${ }^{24} 25$

Figure $3 \Downarrow$ illustrates the importance of a low cognitive function with respect to the later risk of a mild traumatic brain injury. On average, men in the lowest $10 \%$ of overall cognitive function reached a specified number of mild traumatic brain injuries 12 years earlier than did men in the highest $10 \%$ of overall cognitive function. Given this result, evaluation of any environmental factor with the potential to increase cognitive function (such as education) would be of interest. ${ }^{26}{ }^{27}$ In this study, men with a university education had about a $45 \%$ lower risk of a mild traumatic brain injury during follow-up compared with men with elementary school education only, after adjustment for cognitive function and the other identified risk factors.
Other strong independent risk factors for mild traumatic brain injuries included factors related to a low socioeconomic status, such as early disability pension and low total income, previous mild traumatic brain injuries, and intoxications. Young men with low cognitive function, education, and social status are thus at greater risk of mild traumatic brain injuries, sometimes influenced by substance abuse. A different group at high risk seems to include young men with a high physical fitness, which may reflect injuries sustained in sports such as soccer and ice hockey.

The mechanism underlying the association between low cognitive function and mild traumatic brain injuries is of interest. General intelligence includes executive control functions and perceptual speed, two factors that have also been found to be lower after a mild traumatic brain injury. ${ }^{79}{ }^{10}$ Impaired executive control functions include deficits in planning, judgment, and problem solving. ${ }^{28}$ Thus, many factors related to cognitive function can influence the risk of falling and the ability to avoid injuries.

\section{Strength and limitations of study}

This study has several limitations that should be recognised. We evaluated only younger men, so our results may not be applicable to women or older men. We searched in national registers for men diagnosed as having mild traumatic brain injury, and we had no means of validating these diagnoses to exclude more severe traumatic brain injuries. However, 94\% of men diagnosed as having mild traumatic brain injury had a hospital stay of at most two days, which may support a correct diagnosis in most cases. Furthermore, the criteria for inpatient care of a mild traumatic brain injury could have changed during follow-up, and men treated as outpatients were not captured in the registers we used until 2001. Nevertheless,

misclassifications, effects of different diagnostic criteria, or effects of different criteria for inpatient care would probably reduce any differences between men diagnosed as having mild traumatic brain injury and the rest of the cohort towards zero. Strengths of the study include the large well characterised nationwide cohort studied including more than 16000 diagnosed mild traumatic brain injuries, increasing external validity.

\section{Conclusion}

The results of this study suggest that low cognitive function is a risk factor for a later mild traumatic brain injury in the male general population. Other prospective risk factors for mild traumatic brain injuries included low education, low socioeconomic status, intoxications, and high physical fitness. Successful prevention of such injuries may include an assessment and evaluation of these risk factors.

Contributors: PN and AN developed the idea for the study. PN compiled and analysed the study estimates with help and input from AN, SL, and BBE. AN made initial drafts of tables and figures with input from PN, $\mathrm{SL}$, and BBE. AN led the writing of the paper, with contributions from all other authors. $\mathrm{PN}$ is the guarantor.

Funding: The study was funded by the Swedish Research Council. The researchers conducted this study totally independently of the funding body.

Competing interests: All authors have completed the ICMJE uniform disclosure form at www.icmje.org/coi_disclosure.pdf (available on request from the corresponding author) and declare: support from the Swedish Research Council for the submitted work; no financial relationships with any organisations that might have an interest in the 


\section{What is already known on this topic}

Previous retrospective studies have found lower cognitive function, including attention, working memory, episodic memory, verbal learning, and processing speed, after a mild traumatic brain injury

Low cognitive function may be a risk factor for severe traumatic head injuries, but whether it is also a risk factor for the less severe but much more common mild traumatic brain injury is not known

Prospective risk factors for mild traumatic brain injuries identified in population based cohorts are lacking

\section{What this study adds}

Low cognitive function was found in men who later sustained mild traumatic brain injuries

Low cognitive function may be a risk factor rather than the long term consequence of mild traumatic brain injuries

Other prospective risk factors for mild traumatic brain injuries included low education, low socioeconomic status, intoxications, and high physical fitness

submitted work in the previous three years; no other relationships or activities that could appear to have influenced the submitted work.

Ethical approval: This study was approved by the regional ethics board in Umeå and by the National Board of Health and Welfare in Sweden. Data sharing: No additional data available.

1 Murray C, Lopez A. Global health statistics: a compendium of incidence, prevalence and mortality estimates for over 200 conditions. Harvard School of Public Health, 1996.

2 Hyder AA, Wunderlich CA, Puvanachandra P, Gururaj G, Kobusingye OC. The impact of traumatic brain injuries: a global perspective. NeuroRehabilitation 2007;22:341-53.

3 Borg J, Holm L, Cassidy JD, Peloso PM, Carroll LJ, von Holst H, et al. Diagnostic procedures in mild traumatic brain injury: results of the WHO Collaborating Centre Task Force on Mild Traumatic Brain Injury. J Rehabil Med 2004(43 suppl):61-75.

4 Holm L, Cassidy JD, Carroll LJ, Borg J. Summary of the WHO Collaborating Centre for Neurotrauma Task Force on Mild Traumatic Brain Injury. J Rehabil Med 2005:37:137-41.

5 Alexander MP. Mild traumatic brain injury: pathophysiology, natural history, and clinical management. Neurology 1995;45:1253-60.

6 National Institute of Neurological Disorders and Stroke. Traumatic brain injury: hope through research. National Institutes of Health, 2002. (NIH Publication No. 02-158.)

7 Vanderploeg RD, Curtiss G, Belanger HG. Long-term neuropsychological outcomes following mild traumatic brain injury. J Int Neuropsychol Soc 2005;11:228-36.

8 National Center for Injury Prevention and Control. Report to Congress on mild traumatic brain injury in the United States: steps to prevent a serious public health problem. Centers for Disease Control and Prevention, 2003.

9 Wrightson P, Gronwall D. Time off work and symptoms after minor head injury. Injury 1981;12:445-54.

10 Johansson B, Berglund $\mathrm{P}$, Ronnback L. Mental fatigue and impaired information processing after mild and moderate traumatic brain injury. Brain Inj 2009;23:1027-40.

11 Ashman TA, Gordon WA, Cantor JB, Hibbard MR. Neurobehavioral consequences of traumatic brain injury. Mt Sinai J Med 2006;73:999-1005

12 McAllister TW, Flashman LA, McDonald BC, Saykin AJ. Mechanisms of working memory dysfunction after mild and moderate TBI: evidence from functional MRI and neurogenetics. Neurotrauma 2006;23:1450-67.

13 MacDonald CL, Johnson AM, Cooper D, Nelson EC, Werner NJ, Shimony JS, et al. Detection of blast-related traumatic brain injury in U.S. military personnel. N Engl J Med 2011;364:2091-100.

14 Nordstrom A, Nordstrom P. Cognitive performance in late adolescence and the subsequent risk of subdural hematoma: an observational study of a prospective nationwide cohor. PLoS Med 2011;8:e1001151.

15 Andreasson S, Allebeck P, Romelsjo A. Alcohol and mortality among young men: longitudinal study of Swedish conscripts. Br Med J (Clin Res Ed) 1988;296:1021-5.
16 Andersen LB. A maximal cycle exercise protocol to predict maximal oxygen uptake. Scand J Med Sci Sports 1995;5:143-6.

17 Carlstedt B. Cognitive abilities-aspects of stucture, process and measurement. University of Gothenburg, 2000.

18 Carlstedt B, Mårdberg B. Construct validity of the Swedish enlistment battery. Scand J Psychol 1993;34:353-62.

19 Carlstedt B, Gustafsson JE. Construct validation of the Swedish scholastic aptitude test by means of the Swedish enlistment battery. Scand J Psychol 2005;46:31-42.

20 Deary IJ, Strand S, Smith P, Fernandes C. Intelligence and educational achievement. Intelligence 2007;35:13-21.

21 Ludvigsson JF, Andersson E, Ekbom A, Feychting M, Kim JL, Reuterwall C, et al. Externa review and validation of the Swedish national inpatient register. BMC Public Health 2011;11:450.

22 Binder LM, Rohling ML, Larrabee GJ. A review of mild head trauma. Part I: Meta-analytic review of neuropsychological studies. J Clin Exp Neuropsychol 1997;19:421-31.

23 Davies G, Tenesa A, Payton A, Yang J, Harris SE, Liewald D, et al. Genome-wide association studies establish that human intelligence is highly heritable and polygenic. Mol Psychiatry 2011;16:996-1005.

24 Gilbertson MW, Paulus LA, Williston SK, Gurvits TV, Lasko NB, Pitman RK, et al. Neurocognitive function in monozygotic twins discordant for combat exposure: relationship to posttraumatic stress disorder. J Abnorm Psychol 2006;115:484-95.

25 Pitman RK, Gilbertson MW, Gurvits TV, May FS, Lasko NB, Metzger LJ, et al. Clarifying the origin of biological abnormalities in PTSD through the study of identical twins discordant for combat exposure. Ann N Y Acad Sci 2006;1071:242-54.

26 Brinch C, Galloway T. Schooling in adolescence raises IQ scores. Proc Natl Acad Sci U $S$ A 2012;109:425-30.

27 Heckman JJ. Skill formation and the economics of investing in disadvantaged children. Science 2006;312:1900-2.

28 Royall DR, Lauterbach EC, Cummings JL, Reeve A, Rummans TA, Kaufer DI, et al. Executive control function: a review of its promise and challenges for clinical research. $A$ report from the Committee on Research of the American Neuropsychiatric Association. J Neuropsychiatry Clin Neurosci 2002;14:377-405.

Accepted: 18 January 2013

\section{Cite this as: BMJ 2013;346:f723}

This is an open-access article distributed under the terms of the Creative Commons Attribution Non-commercial License, which permits use, distribution, and reproduction in any medium, provided the original work is properly cited, the use is non commercial and is otherwise in compliance with the license. See: http://creativecommons.org/licenses/bync/2.0/ and http://creativecommons.org/licenses/by-nc/2.0/legalcode. 


\section{Tables}

Table 1| Characteristics at baseline of $\mathbf{3 0 5} \mathbf{8 8 5}$ men based on mild traumatic brain injury status during follow-up. Values are numbers (percentages) unless stated otherwise

\begin{tabular}{|c|c|c|c|c|}
\hline \multirow[b]{2}{*}{ Characteristic } & \multicolumn{4}{|c|}{ No of mild traumatic brain injuries } \\
\hline & 1 retrospective $(n=4713)$ & 1 prospective $(n=11217)$ & $>1$ prospective $(n=795)$ & None $(n=288551)$ \\
\hline Mean (SD) age (years) & $18.3(0.7)$ & $18.3(0.7)^{*}$ & $18.3(0.6)$ & $18.3(0.8)$ \\
\hline Mean (SD) weight $(\mathrm{kg})$ & $71.3(10.7)$ & $71.1(11.0)$ & $71.0(11.2)$ & $71.2(10.9)$ \\
\hline Mean (SD) height (cm) & $179(7)$ & $179(7)$ & $178(8)$ & $179(7) \dagger$ \\
\hline Mean (SD) physical fitness (W) & $300(53)$ & $302(53)$ & $297(52)$ & $301(51)$ \\
\hline \multicolumn{5}{|l|}{ Highest achieved education: } \\
\hline Elementary school only & $455(10.0)$ & $1281(11.8)$ & $154(19.8)$ & $20450(7.4)$ \\
\hline 2 years of high school & $1249(27.7)$ & $3611(33.0)$ & $287(36.9)$ & $71840(25.8)$ \\
\hline 3 years of high school & $1400(30.4)$ & $3041(27.7)$ & $219(28.2)$ & $76366(27.4)$ \\
\hline University education & $1450(31.8)$ & 3025 (27.6) & $177(15.1)$ & $110252(39.5) \ddagger$ \\
\hline \multicolumn{5}{|l|}{ Diagnoses at baseline: } \\
\hline Neurosis & $39(0.8)$ & $140(1.2)$ & $15(1.9)$ & $2923(1.0)$ \\
\hline Personality disorder & $12(0.3)$ & $33(0.3)^{*}$ & $3(0.4)$ & $381(0.1)$ \\
\hline Impaired hearing & $606(12.9)^{\star}$ & $1337(11.9)$ & $113(14.2)^{\star}$ & $32357(11.2)$ \\
\hline Impaired vision & $59(1.3)$ & $142(1.3)$ & $19(2.4) \S$ & $2978(1.0)$ \\
\hline Acute intoxication during follow-up & $257(5.5)$ & $1250(11.2)$ & $242(30.4)$ & $7513(2.6) \ddagger$ \\
\hline Early disability income & $84(1.8)$ & $294(2.7)$ & $58(7.4)$ & $3219(1.1) \ddagger$ \\
\hline Mean total income (10 000 SEK) & 24.4 & 22.5 & 16.4 & $25.3 \ddagger$ \\
\hline
\end{tabular}

Information about total income, disability pension, and highest achieved education was collected 14 years after baseline.

*Significantly different compared with group with no mild traumatic brain injuries $(P<0.01)$

†All groups significantly different except when comparing those with one prospective and one retrospective mild traumatic brain injury $(P<0.01)$.

$\ddagger$ All groups significantly different $(P<0.01)$

$\S S$ ignificantly different from all other groups. 
Table 2| Cognitive performance and education in twin pairs where only one twin $(n=63)$ had mild traumatic brain injury before cognitive testing, with men in total cohort with no mild traumatic brain injury shown for comparison

\begin{tabular}{|c|c|c|c|c|}
\hline & \multirow[b]{2}{*}{$\begin{array}{l}\text { Men with no mild traumatic } \\
\text { brain injury ( } \mathrm{n}=288551)\end{array}$} & \multicolumn{3}{|c|}{ Twin pairs discordant for retrospective mild traumatic brain injury } \\
\hline & & $\begin{array}{l}\text { No mild traumatic brain injury } \\
\qquad(n=63)\end{array}$ & Correlation $(r)^{*}$ & Mild traumatic brain injury $(n=63)$ \\
\hline \multicolumn{5}{|c|}{ Cognitive function-mean (SD) } \\
\hline Overall function & $21.3(5.2)$ & $20.1(5.7)$ & 0.79 & $19.7(5.8)$ \\
\hline Logical function & $22.1(5.8)$ & $19.9(6.5) \dagger$ & 0.69 & $19.6(5.9) \dagger$ \\
\hline Word recollection & $18.3(5.1)$ & $17.1(5.7)$ & 0.85 & $17.3(5.7)$ \\
\hline Visuospatial function & $21.4(5.6)$ & $20.1(6.6)$ & 0.75 & $20.6(5.8)$ \\
\hline Technical function & $19.3(4.9)$ & $18.0(4.0) \dagger$ & 0.61 & $17.6(5.4) \dagger$ \\
\hline \multicolumn{5}{|l|}{ Education-No (\%) } \\
\hline Elementary school & $20450(7.4)$ & $7(12)$ & - & $3(5)$ \\
\hline 2 years secondary school & $71840(25.8)$ & $11(18)$ & - & $12(20)$ \\
\hline 3 years high school & $76366(27.4)$ & $15(25)$ & - & $27(44)$ \\
\hline University education & $110252(39.5)$ & $27(45)$ & - & $19(31)$ \\
\hline
\end{tabular}

*Bivariate correlations within twin pairs for different tests of cognitive function.

†Significantly different compared with men with no mild traumatic brain injury in total cohort $(\mathrm{P}<0.05)$. 


\section{Figures}
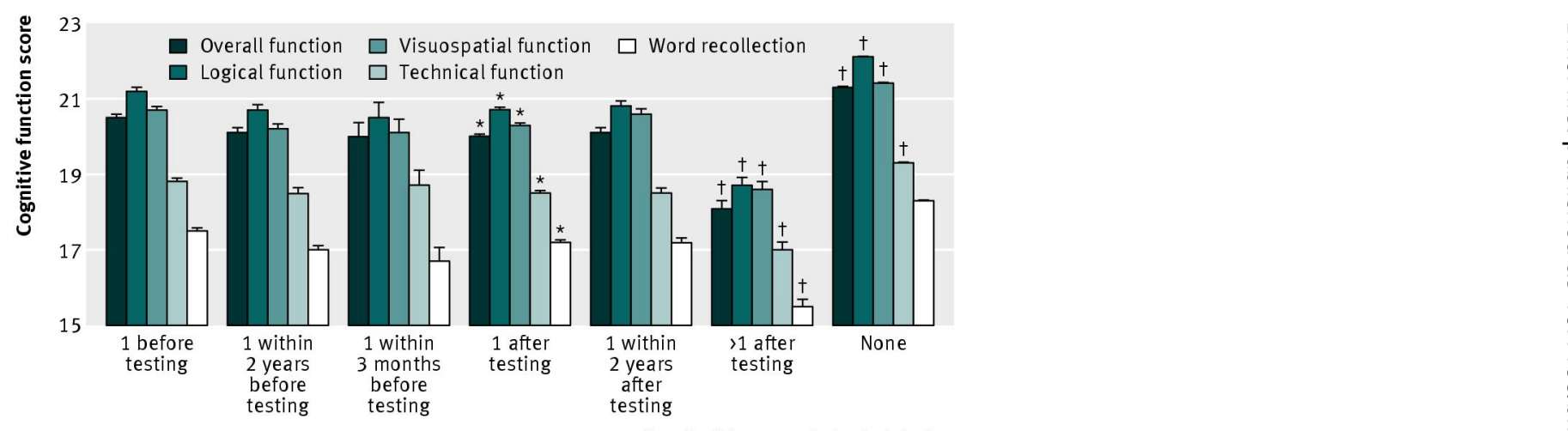

Fig 1 Cognitive function at conscription for total cohort based on mild traumatic brain injury status at follow-up. Means and standard error of means are shown for men with mild traumatic brain injuries before and after cognitive testing at conscription and for those with no such injuries. *Significantly lower $(\mathrm{P}<0.01)$ for specific test compared with men with one mild traumatic brain injury before cognitive testing $(n=4713)$. †Significantly different $(P<0.01)$ for specific cognitive test compared with all other groups 

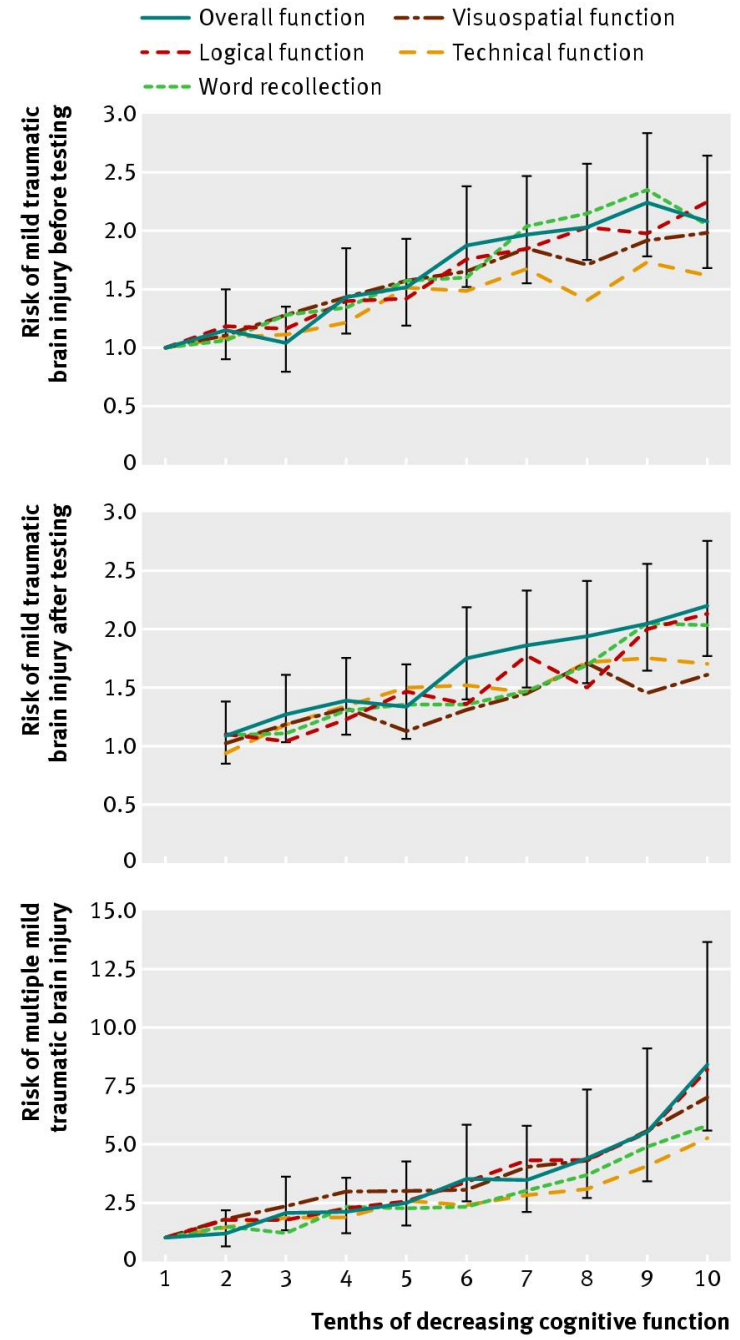

Fig 2 Associations between different estimates of cognitive function and mild traumatic brain injuries. Risk ratios were calculated for each tenth of different measures of cognitive function for men who sustained one mild traumatic brain injury before cognitive testing (top panel), one such injury after cognitive testing (middle panel), or multiple injuries after cognitive testing (bottom panel). The highest tenth of cognitive function was used as a reference for all risk ratio estimations. Associations were adjusted for age, weight, height, vision, hearing, physical fitness, place and year of conscription, and all diagnoses at baseline as listed in table $1 \Downarrow$. 95\% confidence intervals are shown for overall cognitive function

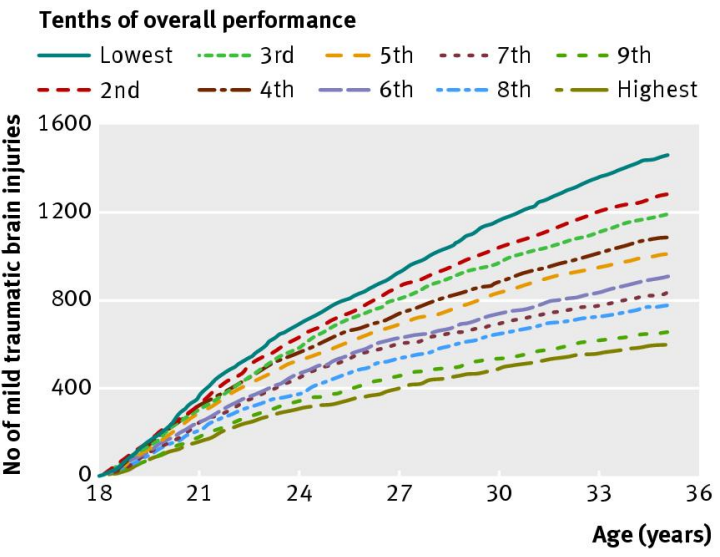

Fig 3 Cumulative incidence of mild traumatic brain injuries for different tenths of overall cognitive function 